\title{
À chacun sa biodiversité
}

Approches savantes et locales de la gestion des ressources et des milieux dans le Sud de la France et le Haut Atlas marocain

To Each His/Her Biodiversity: Local and specialist views of biodiversity and natural resource management

\section{Chantal Aspe et Didier Genin}

\section{(2) OpenEdition}

\section{Journals}

Édition électronique

URL : http://journals.openedition.org/ethnoecologie/1707

DOI : $10.4000 /$ ethnoecologie. 1707

ISSN : 2267-2419

\section{Éditeur}

Laboratoire Eco-anthropologie et Ethnobiologie

\section{Référence électronique}

Chantal Aspe et Didier Genin, «À chacun sa biodiversité », Revue d'ethnoécologie [En ligne], 5 | 2014

mis en ligne le 30 juin 2014, consulté le 03 mai 2019. URL : http://journals.openedition.org/

ethnoecologie/1707; DOI : 10.4000/ethnoecologie.1707

\section{Ce document a été généré automatiquement le 3 mai 2019.}

\section{c) (i) $९$}

Revue d'ethnoécologie est mis à disposition selon les termes de la licence Creative Commons Attribution - Pas d'Utilisation Commerciale - Pas de Modification 4.0 International. 


\section{À chacun sa biodiversité}

Approches savantes et locales de la gestion des ressources et des milieux dans le Sud de la France et le Haut Atlas marocain

To Each His/Her Biodiversity: Local and specialist views of biodiversity and natural resource management

Chantal Aspe et Didier Genin

\section{Introduction}

1 Les termes « Biodiversité » et « Gestion des ressources naturelles » ont progressivement pénétré ces dernières années l'ensemble des discours sociaux en corrélation avec le processus d'institutionnalisation d'une politique de « développement durable » (Aubertin \& Vivien 2005). Ils témoignent aussi d'une globalisation de la problématique écologique qui envisage les problèmes environnementaux comme résultant à la fois de facteurs naturels et sociaux. Mais au-delà des mots, ces acceptions et les pratiques qui s'y réfèrent, recouvrent des réalités perçues parfois de manières très hétérogènes et qui relèvent de systèmes de représentations propres aux acteurs concernés. L'utilisation de ces termes et des significations qu'ils recouvrent, n'est plus l'apanage des seuls milieux scientifiques qui les ont générés sémantiquement. De plus en plus, les populations locales revendiquent des "points de vue » et des «actions» au nom de la biodiversité et de la gestion des ressources naturelles, relayés par tout un courant de recherches sur les savoirs et savoir-faire locaux. Dès lors il apparaît important de mieux cerner les caractéristiques, les points de blocage et les points de convergences possibles entre approches savantes et populaires de la gestion des ressources naturelles et de la biodiversité, pour envisager des concertations et des médiations efficaces et éviter des formes d'action non partagées.

2 Nous nous attacherons ici à montrer comment ces deux types d'approches se différencient, notamment en ce qui concerne la perception et le façonnage des territoires, et l'appréhension de la biodiversité. L'analyse des discours et représentations des approches savantes et des populations locales proposée dans cet article, se base sur les 
résultats de plusieurs recherches interdisciplinaires menées sur les territoires du Luberon, Queyras et Haut Atlas marocain ${ }^{1}$ depuis une dizaine d'années, qui ont mobilisé la combinaison d'enquêtes sociologiques sur les perceptions et de mesures écologiques de terrain.

3 Ces sites sont intéressants à aborder conjointement car ils reflètent une certaine diversité d'approches et de contextes: des Parcs Naturels Régionaux censés mettre au cœur de leurs problématiques les interactions société/environnement, dans les deux premiers cas, une gestion officielle, centralisée et peu participative des espaces naturels par les Services forestiers de l'État dans le troisième; des populations locales diverses et ayant des niveaux de dépendance directe vis-à-vis des ressources naturelles contrastés: montagnardes et très rurales dans les deux derniers cas, plus «urbaines" dans le premier.

\section{Nouvelles représentations des rapports hommes- natures au prisme des savoirs locaux : innovations ou continuités?}

\section{De la « gestion de la nature » à la « gestion de la biodiversité »}

4 Depuis l'apparition, puis l'institutionnalisation de la question environnementale à partir des années 1970, une constante apparaît dans les discours et les pratiques : la nécessité de penser «localement » toute action visant à un développement durable, c'est-à-dire qui satisfasse à la fois les besoins humains sans pour autant mettre en danger les équilibres écologiques.

5 C'est principalement en milieu rural que sont nées des initiatives, d'abord alternatives puis relayées par les politiques publiques, afin d'envisager des normes de conduite des territoires socialement et écologiquement acceptables. En France, ce sont principalement les structures des Parcs Naturels Régionaux qui ont la charge de concilier développement économique local et préservation des ressources naturelles. Pour ce faire, ils établissent des chartes en collaboration avec les acteurs locaux, fixant des objectifs partagés à atteindre.

6 Un nouveau terme s'est progressivement imposé pour désigner cet ensemble de ressources naturelles, celui de biodiversité. Apparu au milieu des années 1980, le concept de biodiversité connaîtra à partir de là un engouement qui dépasse le seul intérêt des sciences de la nature. Le terme se substitue souvent à ceux de "nature» et de « ressources naturelles ». Pour certains auteurs, l'introduction du terme fonde même un nouveau paradigme qui permet en quelque sorte de "réintroduire» l'homme dans l'écosystème et ne plus le considérer seulement comme un perturbateur mais aussi comme producteur de celui-ci :

«Le concept de biodiversité devient alors porteur d'une nouvelle manière de considérer les relations entre sciences de la nature et sciences de l'homme et de la société, ce que j'appellerai un nouveau paradigme. Le fait d'associer étroitement l'homme et la biodiversité dans une même construction globale nécessite de réfuter l'antagonisme traditionnel entre activités humaines et conservation de la biodiversité, cet antagonisme étant par exemple à l'origine de la création des réserves et des parcs: on crée des réserves pour protéger la nature contre l'homme. » (Blondel 2003, p. 17). 
7 La biodiversité et les territoires qui la supportent apparaissent aussi comme les produits historiques de pratiques sociales (espèces domestiques, espaces pastoraux et pastoralisme, forêts rurales et sylviculture,...) (Persha et al. 2011). Le patrimoine naturel ne se conçoit plus uniquement en référence à des éléments déconnectés de l'homme, et la nature primitive ou inviolée ne constitue plus la norme unique de la justification du geste protecteur ou patrimonial. Certaines ressources emblématiques (huile d'argan au Maroc, mélèze dans les Alpes du Sud, par exemple) des paysages sont ainsi perçues comme le résultat d'une longue trajectoire historique d'interactions entre les sociétés locales et leur environnement impliquant des processus complexes d'adaptation, de domestication, de résilience, de sélection, de façonnage des individus et des milieux, ou encore de gestion des risques.

8 Les liens tissés entre paysages, ressources naturelles et sociétés locales, les savoirs et savoir-faire locaux, les empreintes positives et négatives qu'ils ont sur les écosystèmes et à la place qu'ils ont ou devraient avoir dans l'élaboration de stratégies de conservation de la biodiversité sont mis en avant. Les travaux concernant les savoirs locaux ont fait l'objet depuis une vingtaine d'années de nombreuses publications dont certaines constituent des synthèses de référence (Gadgil et al. 1993 ; Berkes et al. 2000 ; Roué 2012 ; Collectif 2005 ; Collectif 2010). Ils montrent pour la plupart que si la biodiversité est un enjeu majeur pour l'humanité, sa conservation ne peut s'envisager que dans la compréhension des mécanismes de sa genèse, de son fonctionnement et de ses interactions avec les sociétés humaines qui tirent avantage de ses nombreux services écologiques mais aussi culturels et socioéconomiques. Ces nouvelles perspectives conduisent progressivement à considérer l'ensemble du processus comme un «patrimoine» qu'il convient de considérer comme un tout et de préserver.

\section{L'émergence de la « patrimonialisation »}

Même si le concept de patrimoine reste largement polysémique et ambigu, nous nous en tiendrons ici à la définition de Ollagnon (2000, p. 339) c'est-à-dire un « Ensemble d'éléments matériels et immatériels, centré sur un titulaire [un individu ou une communauté humaine], qui contribue à maintenir et à développer son identité et son autonomie dans le temps et dans l'espace en contexte évolutif». Ce sont donc bien les propriétés d'identité, de qualité et de transmission de la notion de patrimoine qui en constituent son ossature. Appliquée aux ressources naturelles et à la biodiversité, elle prend un sens éthique au niveau de l'Humanité toute entière, qui introduit l'intérêt et la nécessité de patrimonialiser la Nature (Cormier-Salem et al. 2002). La valorisation qui accompagne les processus de patrimonialisation interroge ainsi les différentes conceptions de la valeur qui soustendent les actions menées au nom de la biodiversité. Avec l'introduction de ce nouveau paradigme apparaissent de nouvelles catégories d'acteurs "concernés ». Ces catégories sont porteuses d'enjeux et d'intérêts qui émergent dans le débat public et peuvent entrer en tension, sinon en conflit, car: "La biodiversité n'a pas la même signification pour des populations dont les systèmes de production et la culture reposent sur un écosystème, pour des firmes pharmaceutiques à la recherche d'une nouvelle molécule ou pour des écologistes soucieux de la préservation d'une espèce animale » (Aubertin et al. 1998, p. 10)

10 Se pose donc le problème de système de valeurs reconnu et érigé en nouvelles normes à respecter. Ces nouvelles valeurs conduisent à construire une nouvelle grille de références qui participe à l'élaboration de représentations considérées comme légitimes et qui 
peuvent entraîner des conflits entre, par exemple, ce qu'est la diversité pour une population locale, qui raisonne à une échelle principalement locale, et la diversité pour une communauté scientifique qui vise à la généralisation de ses savoirs.

Les savoirs naturalistes locaux sont de plus en plus considérés comme des éléments des patrimoines locaux, qu'il convient de conserver et de valoriser au même titre que les diverses composantes de la biodiversité (Berkes \& Davidson-Hunt 2006). Dans le discours international, ils sont successivement passés du statut de connaissances à celui d'outils de gestion, puis à celui d'objets de conservation. Aujourd'hui ces savoirs et les pratiques qui leur sont associées tendent à être considérés comme éléments essentiels des patrimoines locaux ou nationaux, au même titre que les espèces rares ou « emblématiques » (CornierSalem et al. 2002).

Pourtant, dans la pratique, savoirs naturalistes scientifiques et populaires ne s'accordent pas nécessairement sur les politiques de gestion à mener. Les premiers s'appuyant sur des formes de connaissances savantes encore très marquées par la beauté de "la nature naturelle » et la préservation d'espèces emblématiques, les autres plutôt guidés par des connaissances itératives pragmatiques qui ont conduit souvent de manière involontaire et comme conséquences de rapports sociaux spécifiques, à des écosystèmes singuliers (Pinton 2002). Ces deux approches révèlent les difficultés d'interpénétration de formes de pensée forgées sur des modes d'appréhension compréhensive de la réalité différents. $\mathrm{Si}$ bien que parallèlement aux déclarations de principe internationales, qui prétendent à la nécessité d'associer les populations locales aux choix de gestion, persiste un mouvement scientifique qui considère cette alternative comme non concluante :

«Dans le contexte actuel de crise du multilatéralisme, certains s'interrogent sur l'utilité de telles négociations et parlent de revenir à des conceptions plus simples de la conservation, qui prônent un retour à des mesures réglementaires excluant de facto ou de jure toute intervention humaine sur la nature. » (Collectif 2010, p. 7)

Pourtant de nombreuses études (Gadgil 2005; Roué \& Nakashima 2005) montrent combien les connaissances pragmatiques, expérimentales, locales peuvent informer, voire remettre en question les modèles prédictifs des scientifiques à propos des dynamiques des systèmes complexes.

Le problème des modes de décision et de leur légitimité pour imposer les normes de conduite de gestion sont au cœur des débats contemporains. Ils constituent l'enjeu essentiel dans la construction environnementale des territoires soit dans leur homogénéisation soit à l'inverse dans leur diversification.

\section{Normalisation de la territorialisation environnementale et diversité d'approches de la biodiversité}

\section{Le zonage comme élément de différenciation des pratiques et de régulation des usages des ressources naturelles}

15 Il semble que soit reconnue aujourd'hui, si ce n'est l'interaction, du moins l'existence de différentes formes de connaissances, englobant les savoirs, les savoir-faire et pratiques en matière de gestion de l'environnement. Dans la réalité, les deux univers de connaissances des scientifiques et des populations locales ont que trop rarement l'occasion de se côtoyer et s'expriment aussi très souvent dans des rationalités à finalités différentes. Les 
populations locales essaient d'exploiter et gérer leurs ressources dans un but «utilitaire", les scientifiques, quant à eux, cherchent à comprendre les logiques de fonctionnement des gènes, espèces, écosystèmes,...en vue, d'une part, d'alimenter les connaissances d'un champ disciplinaire, lui-même source de notoriété et de pouvoir, et d'autre part, de viser à l'universalisme de ces découvertes.

C'est le plus souvent au sein de structures chargées de mettre en place les politiques publiques environnementales que ces deux catégories d'acteurs sont sollicitées, même si les formes de leur participation restent très variables. Les Parcs Naturels Régionaux en sont un très bon exemple. La finalité des Parcs Naturels Régionaux peut se résumer en grande partie en une conciliation entre les différentes " attentes » sociales en matière de paysage : naturelles, esthétiques, économiques, récréatives. Les paysages ruraux retenus sont présentés comme le résultat d'une relation harmonieuse entre les «hommes et un territoire", entre des données naturelles et des contextes économiques, sociaux et culturels, qui peuvent servir de modèle pour l'avenir.

Pour « révéler » ce paysage, les PNR ont en fait la charge de rationaliser par des normes et actions et rendre opérationnels les attentes de plusieurs catégories d'acteurs : celles des scientifiques, celles des populations locales, celles des extérieurs au territoire (touristes) et surtout celles des représentants des politiques publiques. Dans cette perspective et conformément aux attentes des instances ministérielles (qui approuvent ou non la charte et renouvelle ou non le label «Parc »), les PNR vont proposer un zonage de leur territoire, qui à la fois délimitera les frontières de l'action ainsi que les types d'actions à mener.

Analysons deux cas dans le Sud-Est de la France, celui du PNR du Luberon, à cheval sur deux départements, le Vaucluse et les Alpes de Haute-Provence, et qui comprend 85 communes concernant près de 170000 habitants, et celui du Queyras, situé dans les Hautes-Alpes englobant huit communes et le territoire «non habité » de deux autres communes représentant autour de 2300 habitants. Le premier s'étend sur 175000 ha avec une densité de $84 \mathrm{hab} / \mathrm{km}^{2}$, et le second sur 65000 ha pour une densité de $3 \mathrm{hab} / \mathrm{km}^{2}$.

\section{Les zonages des PNR conformes aux attentes scientifiques et aux politiques publiques}

Les logiques de découpages du territoire sur lesquels vont porter des politiques d'action différenciées varient assez sensiblement au niveau des deux PNR. Le PNR Luberon met en avant de manière prioritaire un zonage ayant des caractéristiques proches des canons d'appréciation de l'écologie scientifique, alors que celui du Queyras se présente comme une mise en conformité avec les directives et textes nationaux et internationaux.

Les zones proposées par la charte du PNR Luberon sont les suivantes (PNR Luberon, Charte, Objectif 2021, p. 29-35):

«-Zone de nature et de silence :

Définie depuis la création du Parc, elle couvre les espaces inhabités du massif du Luberon, des versants sud des Monts de Vaucluse et des collines des bords de Durance au sud et à l'est. L'objectif est d'y préserver l'authenticité d'un rapport de l'homme à la montagne basé sur des pratiques non banalisantes et respectueuses des milieux naturels, de la faune et de la flore, des paysages et des usages traditionnels agricoles, pastoraux, forestiers, cynégétiques, de cueillette et de loisir...

- Les Secteurs de Valeur Biologique Majeure :

Dès la genèse du Parc et avec l'appui de son Conseil Scientifique, un travail 
d'inventaire cartographié des richesses naturelles du territoire sous l'intitulé « Secteurs de Valeur Biologique Majeure "a consisté à cerner les grandes formations végétales originales.

Les pourtours de ces secteurs ont ensuite été affinés à la lecture de la diversité biologique, floristique et faunistique qui les caractérisent ainsi que par la fonctionnalité des écosystèmes...

- Les milieux exceptionnels :

Ils sont délimités à l'intérieur des Secteurs de Valeur Biologique Majeure et font l'objet de mesures de conservation particulières.

- Les espaces ruraux et les terroirs agricoles :

Ces espaces combinent aujourd'hui de façons très différentes sur le territoire des fonctions productives, résidentielles, touristiques et de nature...,

- Les Zones urbanisées..

- Les Unités paysagères... »

21 Le zonage proposé par les rédacteurs de la charte du PNR du Luberon, territoire relativement urbanisé, semble révéler une volonté de définir des zones de nature encore relativement préservée d'intervention humaine et octroie pour cela une place privilégiée à l'approche savante des milieux, comme en témoigne l'intitulé du premier objectif de la charte : «Développer la connaissance scientifique ». (Objectif A.1.1, p. 1)

Les rédacteurs de la charte du PNR du Queyras ont choisi quant à eux une toute autre option qu'ils formalisent de la manière suivante :

«Ainsi le territoire du Parc est pris en compte par l'État, la Région et le Département pour la mise en œuvre expérimentale de leurs politiques ou de celles de l'Europe en matière de protection, d'aménagement du territoire et de développement. Il est considéré comme un lieu privilégié pour la mise en œuvre coordonnée des politiques publiques. »(PNR Queyras, Charte 2010-2022, 2010, p. 5).

Le zonage proposé et cartographié vise à montrer sur quelle portion territoriale doivent s'opérer les mesures réglementaires : Agenda 21 local, directive habitat, directive oiseaux, réserve nationale Ristolas-Mont Viso, arrêtés de biotope, projet de Biosphère...Le soustitre choisi pour présenter la charte est à ce titre assez significatif : "Biosphère, écotourisme et agriculture durable: Queyras, Haute montagne exemplaire ». L'idée principale qui semble présider aux différentes actions proposées telles l'écotourisme, le développement des produits biologiques, de l'artisanat local, la maîtrise de l'urbanisme par le "développement d'une culture de l'urbanisme et des paysages", la mise en place de transports respectueux de l'environnement,..., est celle d'une nature paysagée qui serait celle attendue par les acteurs extérieurs : touristes et instances environnementales.

La présentation rapide de ces deux exemples témoigne de la prégnance forte des discours des « extérieurs au territoire » dans la politique des PNR.

$\mathrm{Au}$ Maroc, les espaces non cultivés, et notamment tous les espaces forestiers, sont considérés comme étant de la propriété et de la compétence exclusive de l'État. Ils sont pourtant intégrés dans des territoires ruraux et exploités dans le cadre de vie souvent très dépendants de l'exploitation des ressources naturelles. Les zonages effectués visent à empêcher l'emprise des populations locales sur ce " patrimoine » national. Ils se situent à différents niveaux d'échelles en fonction des conditions éco-climatiques, des risques environnementaux et des potentialités productives des espaces. Ils se traduisent par des planifications de gestion dans lesquelles les éventuels « utilisateurs » n'ont que peu droit à la parole. Il s'ensuit des gestions officielles et « de fait » qui parfois peuvent générer des expériences intéressantes, mais qui, le plus souvent se manifestent par des conflits 
d'intérêts et de pouvoir entre services étatiques et utilisateurs locaux (Genin \& Benchekroun 2007, Aubert 2010).

\section{Les territoires des populations locales et leurs zonages}

Les populations locales opèrent, elles aussi, des découpages sur leur territoire, mais en procédant à partir de constructions mentales différentes. Celles-ci s'organisent davantage sur des formes de connaissances pragmatiques et/ou liées à des savoirs transmis très souvent oralement, sur des appropriations territoriales liées aux déplacements et au fonctionnement des systèmes de production locaux, aux liens d'interconnaissances ou encore à des constructions historiques. Ces découpages reposent ainsi très souvent sur des formes d'appropriation symbolique liées à la fois à une histoire collective et individuelle dans lesquelles les critères de morphologie géophysique semblent jouer un rôle important.

Selon notre enquête, dans le PNR du Luberon, la montagne du même nom semble être une barrière pour délimiter ce que les individus nomment "mon Luberon ». L'autre côté ce sont « les autres ». Mon territoire correspond à la face du Luberon « que je peux voir » et sur laquelle sont situées « des villages que je connais bien ». Pour ceux situés sur la face sud, le territoire du Luberon est celui qui correspond à l'appellation locale du «pays d'Aigues» et qui regroupent une dizaine de communes ayant historiquement des relations fortes de voisinage, marquées par des échanges économiques et des formes d'exploitation du territoire proches (culture de la vigne et maraîchage). Ceux de la face nord se considèrent comme étant du "pays d'Apt ", marqué par un climat plus rude et davantage en liaison avec l'arrière pays bas-alpin, caractérisé par la culture de la cerise, de la lavande et l'élevage de moutons.

Dans le PNR du Queyras, les frontières géographiques sont encore plus marquées et fortement identitaires. Le territoire du Queyras pour les habitants correspond à celui des huit communes situées autour du bassin versant de la rivière Le Guil et délimité au nord et à l'est par les cols d'Izoard et Agnel et la frontière italienne, au sud et à l'est par le bourg de Guillestre et la commune de Vars, qui pour les Queyrassins, ne font pas à proprement parler partie de leur territoire. Outre les critères géomorphologiques, les justifications évoquées sont celles de l'unicité d'un climat rude qui conditionne des modes de vie et des cultures. Il est d'ailleurs très souvent reproché aux techniciens du parc de ne pas, pour la plupart, habiter dans cet espace et de fuir la vie que mènent les habitants l'année durant.

Que ce soit dans le Luberon ou le Queyras, le partage du territoire se fait en fonction du relief, des paysages et des cultures agricoles associées. Il y aurait en gros quatre types de zones, les espaces cultivés, les espaces pâturés, les espaces habités et les espaces forestiers. La forêt occupe un repère essentiel dans cette construction, elle peut être considérée comme élément de nature "naturelle » à préserver pour les habitants du Luberon ou au contraire comme témoin de la déprise agricole pour ceux du Queyras.

30 À un niveau plus fin, les territoires ruraux sont aussi le plus souvent explicitement compartimentés par les populations locales en espaces-ressources différenciés, fruits d'une longue histoire d'interactions entre, d'une part, les hommes et la nature et, d'autre part, les hommes entre eux. Dans le Queyras, une grande portion du territoire est dédiée à l'élevage. Le zonage opéré localement dépasse les seules conditions topo-écologiques des milieux pour englober leurs fonctions dans les systèmes de pâturage et d'utilisation 
de l'herbe (prairies de fauche précoces ou tardives, alpages, pâturages d'intersaison, zones de soudure, zone de "récréation » pour les animaux, zones du matin, de l'aprèsmidi, etc.). Il correspond à des préoccupations de «fonctions » et « d'insertion » dans un schéma global d'usages mêlant spatialité et temporalité (Petit \& Fleury 2010; Vincent 2011; Genin et al. 2012a) Il reprend aussi les divisions foncières et l'histoire même des hommes et des évènements. Ainsi la toponymie locale est riche d'enseignements sur ces savoirs et interactions tissés au fil du temps: «les prés des neiges de juillet », ou le lieudit «la mère du bon foin" sur la Commune d'Arvieux correspondent aussi à des indications en termes de conduite des troupeaux, d'histoires de vie ou de risques à prendre en compte. Cette richesse linguistique ne concerne pas seulement la description des territoires et, plus globalement, les taxinomies populaires pour nommer les choses de la nature n'ont souvent rien à envier aux classements complexes des scientifiques, elles s'accordent là aussi, le plus souvent, à des fins utilitaires d'exploitation et de gestion des ressources locales (Roué \& Nakashima 2005)

Dans le Haut Atlas marocain, les paysages ruraux de montagne présentent aussi une structuration très marquée avec des zones d'agriculture irriguée en terrasses, en fond de vallée, des espaces sylvopastoraux ayant des couverts forestiers et des physionomies forestières diversifiées aux abords de villages et sur les pentes, et des espaces pastoraux asylvatiques montant jusqu'aux crêtes des montagnes à plus de 3000 mètres d'altitude (Auclair et al. 2011). Cette structuration se retrouve très classiquement dans tout le monde montagnard berbère. Chaque compartiment est décliné en différents espaces intégrant des modes d'utilisation et de gestion divers, dont la mise en agdal est un exemple emblématique. L'agdal est un terme générique d'origine berbère désignant un mode d'appropriation et de gestion de la terre en bien commun mis en œuvre par une institution locale (assemblée villageoise, inter-villageoise ou inter-tribale) qui fixe les droits et règles d'accès et d'usages des ressources naturelles pour en assurer leur renouvellement. Il consiste toujours en une mise en défens pendant des périodes définies et en une définition précise des modalités d'exploitation des ressources (Genin \& Simenel 2011). Dans un des villages que nous avons étudiés dans la haute vallée des Aït Bouguemez (Haut Atlas central), par exemple, les populations locales expliquent très facilement qu'ils ont compartimenté leur espace forestier en différentes zones, lesquelles remplissent des fonctions différenciées et que l'on peut individualiser par leur physionomie (Genin \& Simenel 2011) :

- des zones conduites en agdal, sont ouvertes aux prélèvements uniquement lorsqu'il y a beaucoup de neige en hiver afin d'alimenter les animaux en fourrage foliaire et se procurer du bois pour la chauffe et la cuisine. La période d'ouverture, décidée par la jmaa (assemblée villageoise), peut atteindre plusieurs mois. Un autre agdal constitué uniquement de genévriers rouges (Juniperus phoenicea), non consommé par le bétail, est dédié à la production de perches de faible diamètre, utilisé comme supports transversaux aux poutres dans la fabrication des toitures.

- des zones hors agdal, soumises à des règles d'usages moins contraignantes, sont fortement exploitées pour la coupe de bois, la cueillette de fourrage foliaire et le pâturage. Elles prennent souvent la forme de matorrals hauts et de forêts claires, considérés par les forestiers comme des espaces dégradés et improductifs, mais dont l'intérêt en biens et services est pourtant largement évoqué par les populations locales. Une zone plus éloignée, appelée localement Taghrout $n$ Ait Ayoub, constitué de grands genévriers thurifères est réservée exclusivement à l'exploitation des poutres. 


$$
\begin{aligned}
& \text { des éléments de la biodiversité, et, d'autre part, représentent de véritables « espaces- } \\
& \text { ressources » aux fonctions très différenciées. }
\end{aligned}
$$

formations arborées qui, d'une part, participent à la diversité écosystémique qui est un

\title{
Les représentations scientifiques et populaires de la biodiversité
}

Issu de la sphère scientifique, le concept de biodiversité s'est progressivement propagé à l'ensemble de la société. Le terme n'est de fait pas inconnu des populations locales que nous avons interrogées, même si celui-ci recouvre pour chacun des contenus différents. Il est très souvent associé à une "diversité de la vie», ce qui correspond à une décomposition du terme assez proche de son ancêtre linguistique « diversité biologique ». Barbault, raconte ce passage alors qu'il était jeune chercheur en écologie :

\begin{abstract}
« Au début, quand Francesco di Castri m'a parlé de biodiversité, je n'ai pas rigolé, mais j'ai pensé 'la diversité du vivant, il n'y a rien de nouveau sur la planète'. Mais il savait susciter l'enthousiasme, et de même qu'il m'avait entraîné dans les réserves de biosphère au Mexique, je l'ai suivi sur ce nouveau terrain. Cela a commencé à Rio avec des débats sur la biodiversité. Ce que j'ai découvert, et ce qui m’a intéressé, c'était les questionnements sur la diversité du vivant; les liens entre diversité du vivant et l'homme : cela ouvrait complètement le champ à des approches tout à fait différentes, nouvelles. » (Migot \& Roué 2006, p. 39)
\end{abstract}

À lire ces propos, largement partagés par quelques grands noms de la discipline (Blandin, Blondel), il serait légitime de penser qu'aujourd'hui l'écologie scientifique a intégré dans ses pratiques la prise en compte des interactions entre les différents éléments du vivant, c'est-à-dire la faune, la flore et les êtres humains. Mais la réalité est quelque peu différente et nos enquêtes ont montré que bien souvent les populations locales avaient une approche beaucoup plus systémique que les scientifiques eux-mêmes.

\section{Des représentations scientifiques tributaires des formations académiques : la recherche de lois générales par la sectorisation}

Le propre de toute science est de viser à la généralité de ses propos et de construire des lois universelles du fonctionnement des objets qu'elle étudie. L'écologie n'échappe pas à ce processus et considère son objet comme étant l'écosystème. Concept inventé par Tansley en 1935, il va permettre à cette discipline de prendre son autonomie par rapport à la biologie, en réunifiant ce qui avait été séparé, tout d'abord la faune et la flore (avec le concept de biocénose), puis le vivant avec le milieu dans lequel il vit :

« Au fond, la pensée écosystémique substitue à un espace écologique divisé, un espace écologique réunifié dans lequel les facteurs abiotiques et biotiques de l'environnement ne représentent plus que deux aspects d'une même réalité.» (Acot 1988, p. 134)

Mais les formations académiques restent, pour la grande majorité, assez fermées à cette approche globalisante et découpent toujours leur enseignement en " écologie végétale » et " écologie animale ", puis en sous-parties indépendantes comme l'écophysiologie ou l'éthologie. Les thèses se chargent ensuite de produire d'autres spécialisations, sur le genévrier ou le pin sylvestre, les papillons ou les coléoptères, voire une étape du développement d'une espèce de papillon particulière. Ceci conduit dans la pratique, et la politique de défense de certaines espèces dans les PNR en est un bon exemple, à faire appel aux spécialistes de telle espèce qui considèreront au mieux «l'écosystème » 
nécessaire à son espèce, sans tenir compte de travaux plus interdisciplinaires qui seraient pourtant très pertinents.

La diversité biologique, ou biodiversité, est un concept très large qui rassemble la diversité infraspécifique (variabilité génétique, adaptations des écotypes, sous-espèces et effets de la géographie ou de la phénologie sur la spéciation, etc.), la diversité spécifique (richesse en espèces, taux d'endémisme spécifique, etc.) et la diversité supra-spécifique (richesse et particularisme des genres et des familles, histoire évolutive des phylums, etc.). Il subsiste dans l'appréhension de cette biodiversité spécifique, le problème de l'échelle de perception. Selon que l'on raisonne à l'échelle du monde ou de la station étudiée, la perception de la biodiversité change. Se pose alors un autre niveau de sectorisation lié à l'échelle d'observation.

Si la "diversité génétique " semble aujourd'hui un champ de recherches en plein développement, elle est encore relativement peu mise en pratique dans la gestion des ressources naturelles, du moins dans les cas que nous avons analysés. L'accent est mis principalement sur la conservation d'espèces ou de milieux pris dans leurs entités propres. La biodiversité est souvent considérée de façon fractionnée: il s'agit de conserver une espèce, voire un milieu, mais elle n'est que rarement liée au fonctionnement des systèmes productifs en place. Ainsi il s'agit de «mitiger » les «impacts» en proposant des modes de gestion censés maximiser un ou plusieurs éléments de la biodiversité, pris de manière déconnectée de structures productives opérant sur le territoire et de fonctionnements plus globaux. Par exemple dans la charte du Queyras, au chapitre B2 traitant plus spécifiquement de la biodiversité, on peut lire dans les actions prévues ${ }^{2}$ :

«- Assurer un suivi de l'évolution des espèces rares et endémiques, des milieux, des écosystèmes/assurer une veille environnementale

- Assurer une gestion exemplaire des sites faisant l'objet de classement ou justifiant une qualité emblématique ».

On retrouve des préoccupations similaires dans le PNR du Luberon, avec des propositions du type : «Veiller à l'évolution de Secteurs de Valeur Biologique Majeure », « Renforcer la protection des milieux exceptionnels" "S'impliquer dans la protection des espèces animales et végétales particulièrement menacées ».(Charte PNR Luberon, 2010, p. 43-45-50).

Dans le Haut Atlas des schémas comparables sont mis en avant, notamment avec la définition et la caractérisation de SIBE (Site d'Intérêt Biologique et Écologique) présentant des éléments de "Nature» particuliers (espèces sauvages, paysages) à conserver. Ces initiatives ont permis une prise de conscience et la mise en place d'actions concrètes pour la protection d'espèces emblématiques comme le cèdre de l'Atlas, le mouflon de l'Atlas ou le macaque de barbarie (ou magot), qui s'agit de protéger de l'impact anthropique. Et parallèlement tout un discours s'est développé sur la dégradation anthropique généralisée des milieux naturels, et notamment de la forêt, pour laquelle la législation signifie qu'elle ne peut être gérée que par des services spécialisés de l'État (Davis 2005). Dans les zones de la montagne rurale, la forêt est déclinée par les forestiers en peuplements homogènes - et uniquement en termes de peuplement - selon des critères écologiques (structures de peuplement), de capacité de production de bois et/ ou d'intérêt de protection des sols. Mais les aspects relatifs aux statuts traditionnels (agdals), pourtant très riches de savoirs et savoir-faire relatifs à l'écologie des plantes (Aloui 2009; Auclair \& Alifriqui 2012), ou aux fonctions d'usages pour les populations 
locales, ne sont en aucun cas reconnus comme critères de zonage pertinents, alors que ceux-ci représentent encore dans certaines zones les principaux composants de la gestion locale des milieux et des ressources (Genin et al. 2012b).

Il apparaît difficile pour les gestionnaires de sortir du cloisonnement inhérent à la classification scientifique, car leur principal mode de légitimation repose sur ce qui est du domaine scientifique à propos des choses de la nature. Et force est de constater que ce sont les scientifiques (en oubliant les sciences humaines) que les instances de décisions nationales et internationales convoquent pour lister les espèces ou les milieux à protéger, conserver ou restaurer. Les populations locales ne sont pas encore sollicitées comme experts pourvoyeurs de données "fiables » car trop peu formalisées et considérées comme trop subjectives.

Pourtant, les modes de raisonnement de celles-ci sont souvent plus globalisants que ceux des scientifiques et leurs apprentissages liés à une réalité de terrain les ont souvent conduits à des formes de connaissances de leurs écosystèmes, sans prétention généralisante, mais qui mériteraient sans doute d'être prises en compte.

\section{Des formes de connaissances populaires localisées, fonctionnelles et systémiques}

43 L'élément de raisonnement, sur lequel diffèrent profondément les appréciations scientifiques et populaires, tient à la revendication par les populations locales d'un « particularisme local » dans le fonctionnement de leurs territoires. Aucune recherche de généralisation n'est mise en avant, au contraire elles poussent à l'extrême la revendication localisée de leurs propos. On pourrait résumer cette pensée de la manière suivante : «Ici c'est comme ça. Ailleurs on ne sait pas, mais ce doit être différent ». Les habitants parlent ainsi de ce qu'ils connaissent et qu'ils ont la plupart du temps expérimenté (ou leurs proches, ou leurs ancêtres). Cette expérimentation est particulièrement présente dans le discours des agriculteurs, principaux acteurs gestionnaires des ressources naturelles :

«A un moment donné, ils (les techniciens du Parc), nous ont dit « vous avez eu des primes pour faire ça dans le parc, alors il faut conserver à cet endroit là, il ne faut pas faucher avant telle date " mais à cette date là c'est du foin qu'on peut foutre en l'air, ça ne vaut plus rien. Ils veulent qu'on fauche pas avant le 10 juillet, pour que les graines tombent, pour conserver les fleurs et tout. Mais ça fait des années et des années qu'on fauche et les fleurs sont encore là. Moi j'ai dit, je fauche le $1^{\mathrm{er}}$ juillet. On connaît notre endroit quand même !...En plus, en bas ${ }^{3}$, ils veulent faire manger les bêtes de bonne heure. Nous, on ne peut pas, parce qu'il faut que l'herbe soit un peu dure autrement ça leur donne la diarrhée. Ça je suis aussi arrivé à leur faire comprendre. » (Éleveur, Queyras)

Mais on le retrouve aussi chez d'autres catégories sociales, comme par exemple cet habitant du Luberon, travaillant comme mécanicien :

«J'ai une petite histoire à vous raconter. Près du poulailler, j'avais un petit arbre qui poussait, j'ai cru que c'était un noisetier, et en fait c'était un orme, à l'époque où tous les ormes crevaient. Pourquoi le mien n'a pas été malade? Parce qu'il était entouré d'une haie de lilas, or les écolos ont mis beaucoup de temps à voir que là où il y avait du lilas, les ormes ne crevaient pas. Alors ça faisait rigoler les techniciens. Alors que même à l'INRA, ils ne trouvaient pas, ils cherchaient à isoler le principe qui préserve l'orme, il n'y en a pas un qui a dit mais plantez du lilas au pied de vos ormes, ça pousse comme du chiendent. À moins que ce ne soit spécifique à la terre d'ici. Voilà !» 
L'autre élément notable des modes d'analyse de la biodiversité par les populations locales est leur approche duale de celle-ci : d'une part non sectorielle, systémique pourrait-on dire, avec en quelque sorte une perception linnéenne de la nature, " tout a une fonction ", même si cette fonction est souvent ramenée à l'homme puisqu'il y est question d'« utilité », d'autre part, par rapport à son utilité et aux fonctions qu'elle peut avoir pour les activités humaines d'utilisation des ressources naturelles. Ainsi, on retrouve très classiquement des discours du type :

"Même si on ne comprend pas tout, il est certain que la nature a horreur du vide et que tout ce qui existe dans la nature, que ça a été créé par Dieu ou par autre chose, et bien ça a un sens, une fonction. " (Femme au foyer, Luberon)

«Pour moi, tout ce qui est dans la nature est beau. On est tous lié dans le cadre d'un cosmos. L'être humain a trop voulu faire l'apprenti sorcier, sans se soucier de la liaison qui existe entre lui et le reste de la vie sur la terre. Pourtant, si telle petite plante est là, même si on ne la remarque pas, et ben elle a son utilité. » (Employée commerce, Luberon)

La vision utilitaire et fonctionnaliste est encore plus exacerbée chez les paysans berbères du Haut Atlas (Genin et al. 2012b). Dans les agdals pastoraux, ils fondent leur discours sur la nécessité de favoriser au maximum le développement d'un grand nombre de plantes et leur permettre de "faire la graine ». Ainsi les périodes de fermeture et d'ouverture de l'agdal sont explicitement calées en fonction de l'accomplissement du cycle phénologique de toutes les espèces présentes, comme l'a montré Alaoui (2009). Spontanément les paysans indiquent des plantes intéressantes qu'ils présentent comme spécifiques aux agdals et se développant uniquement grâce à leurs actions de "protection de l'agdal ", citant par exemple Amenzel (Stipa sp.), Ouizra (Arenaria pungens), Tadrt n Ouhchen ( Eryngium sp.), Tiright (Ranuncula sp.). Mais la richesse floristique est toujours perçue en termes d'intérêt ou non pour le bien-être du troupeau, et si elle est perçue comme en général favorable, elle est toujours ramenée à sa fonction positiviste dans la conduite des troupeaux. Ceci est particulièrement mis en évidence lors d'années sèches où certains critiquent la fermeture de l'agdal en argumentant que cette pratique ne permet pas, dans ces cas là, de remplir sa fonction de favoriser le développement de fourrages. Ces milieux pastoraux sont ainsi appréhendés avec une vision "paysagère» globale, faisant intervenir à la fois la richesse floristique, les conformations géo-topographiques, ainsi que les complémentarités et séquentialités d'utilisation des différents espaces. Par contre, le discours est très différent lorsqu'on aborde la thématique de la biodiversité dans les espaces boisés. L'élément le plus frappant que l'on a pu retirer des multiples entretiens effectués auprès des populations locales concernant ces espaces, est que les gens ne se réfèrent qu'aux arbres (Genin et al. 2012b). Les ressources du sous-bois ne sont pas distinguées, considérées seulement comme fourrages «de passage » pour les troupeaux allant pâturer dans des zones plus éloignées du village, et ne faisant pas l'objet de préoccupations particulières. Par contre, tout un discours se développe autour des formes de gestion et d'exploitation des arbres pouvant garantir le renouvellement de la ressource: il s'agit d'émonder les arbres partiellement par coupe des branches périphériques de l'arbre (diamètre jusqu'à $8-10 \mathrm{~cm}$ ). Il est constamment veillé à laisser intactes les branches axiales de manière à ne pas trop gêner la croissance de l'arbre. Certains indiquent que, en général il est respecté un délai de deux années à un arbre avant d'être coupé à nouveau. Le nombre de branches coupées par arbre est variable selon sa taille et son état (6-8 branches en moyenne). 
critères de "vision globale » et " d'utilité " peuvent parfois constituer par contre un point d'achoppement et de conflit fort entre les acteurs concernés par la biodiversité, surtout si celle-ci peut nuire à l'activité de certains. C'est le cas de la réapparition du loup dans les Alpes du sud, qui pose de manière évidente des questions fondamentales de la place à accorder aux activités humaines, et de choix clairs en matière de gestion des espaces et des ressources, comme l'illustrent les propos d'un éleveur du Queyras :

«Quand vous voyez les trucs animaliers, le loup c'est beau, c'est fidèle, il élève bien ses enfants. Mais nos brebis, elles sont autant attachantes. Bon ça va pour la boucherie, mais si on ne les aimait pas, on ne se lèverait pas la nuit, c'est aussi important pour nous que le loup... Au début, on y croyait, on voulait se défendre, expliquer ce que c'est et les inconvénients que ça va faire dans la montagne mais maintenant on ne se bat plus. On sait qu'ils vont revenir en arrière au bout d'un moment quand il y aura trop de loups et tout. Entre temps, il y aura des choses qui se seront perdues et qui se seront dégradées. Peut-être qu'on est en train de foutre quelque chose en l'air. Si ça devient difficile d'élever des moutons, on n'en fera plus, et si on n'en fait plus, les prés ne seront plus fauchés et les buissons envahiront partout. Si c'est ça qu'ils veulent...»

Ainsi, que ce soit au nord ou au sud, la biodiversité, si elle est perçue comme en général favorable, semble toujours être ramenée à sa fonction positiviste dans les usages que l'on peut en faire. On peut alors parler d'une perception fine de la part des populations locales d'une « biodiversité de fonction », qui est intégrée, d'une part, dans un système plus large d'évaluation des milieux et des ressources en termes d'opportunités de pratiques, d'autre part, dans des systèmes de représentations holistes liant différents éléments de nature et l'homme.

\section{Conclusion}

L'introduction de la notion de «gestion de la biodiversité » dans les politiques publiques nationales et internationales, a permis de reconsidérer, au moins au niveau des intentions, l'importance des savoirs locaux dans la planification territoriale et la mise en place de nouvelles normes de gestion. Mais à l'heure où la gouvernance environnementale est mise en avant comme élément fondamental de la gestion des territoires, où participation et concertation sont érigées en outils miracle à appliquer dans toute action en matière de développement territorial et d'usages des ressources, force est de constater que dans la majorité des cas on assiste à des dialogues difficiles entres acteurs dont la place, les compétences et la légitimité sont souvent mal prises en compte. Ceci est dû, à notre sens, à deux éléments majeurs : l'asymétrie des pouvoirs, notamment entre acteurs locaux et institutions publiques, et, en amont, à une méconnaissance mutuelle de la teneur et des modes de construction des savoirs des groupes d'acteurs en présence. En ce sens, la prise en compte des savoirs locaux ne doit pas être cantonnée à une collecte de pratiques décontextualisées de leur environnement social, historique et culturel afin d'en faire rentrer quelques-unes dans un canevas de pensée et d'actions, déjà élaboré selon des canons scientifiques préétablis. De même, les savoirs scientifiques doivent pouvoir être mieux expliqués et appréhendés par les acteurs des territoires concernés, afin d'en exploiter les apports fondamentaux en termes de diagnostic, de prospective et d'éléments d'aide à la décision. Ainsi la co-construction de solutions originales et efficaces en matière de gestion des territoires, des ressources naturelles et de la biodiversité suppose-t-elle avant tout, écoute et partage réels, dans une 
optique de prise en compte des diversités culturelles locales qui éviterait un morcellement des actions en savoirs de spécialistes.

\section{BIBLIOGRAPHIE}

Acot P. 1988 - Histoire de l'écologie. Paris, PUF, 285 p.

Aloui S. 2009 - Les pelouses humides dans le Haut Atlas : biodiversité végétale, dynamique spatiale et pratiques de gestion coutumière. Thèse Doctorat, Écologie végétale et Environnement, Université Cadi Ayyad, Marrakech, Maroc, 120 p.

Aspe C. \& Tatoni T. 2003 - Dynamique de la biodiversité, formes de connaissances et impact des pratiques sociales. LPED/IMEP. Institut Français de la Biodiversité, Rapport de recherche 55 p.+ annexes.

Aubert P.-M. 2010 - Action publique et société rurale dans la gestion des forêts marocaines : changement social et efficacité environnementale. Thèse Doctorat Sci. Soc. de l'environnement, AgroParisTech, Engref, Montpellier, $386 \mathrm{p}$.

Aubertin C, Boisvert V. \& Vivien F.D. 1998 - La construction sociale de la question de la biodiversité. Natures Sciences Sociétés 6(1) : 7-19.

Aubertin C. \& Vivien F.D. 2005 - Le développement durable: enjeux politiques, économiques et sociaux. Paris, La Documentation Française, Paris, IRD, 143 p.

Auclair L., Simenel R., Alifriqui M. \& Michon G. 2010 - Agdal. Les voies imazighen de la patrimonialisation du territoire. Hespéris Tamuda 45 : 129-150.

Berkes F., Colding J. \& Folke C. 2000 - Rediscovery of traditional ecological knowledge as adaptative management. Ecological Applications 10(5) : 1251-1262.

Berkes F. \& Davidson-Hunt I. 2006 - Biodiversité, systèmes de gestion traditionnels et paysages culturels. Revue Internationale des Sciences Sociales 187 : 39-52.

Blandin P. 2009 - De la protection de la nature au pilotage de la biodiversité. Versailles, Quae (Sciences en questions), $122 \mathrm{p}$.

Blandin P. 2010 - Biodiversité. L'avenir du vivant. Paris, Albin Michel, 263 p.

Blondel J. 2003 - Biodiversité, quels enjeux pour les sociétés? In IFB (Ed.) Actes des journées de l'Institut Français de la Biodiversité. Tours 18-20 décembre 2002. Paris, IFB :17-19.

Collectif 2005 - Biodiversité. Conserver pour qui ? Courrier de la Planète 75, Janvier-Mars, 68 p. Collectif 2010 - Biodiversité. Des sciences pour les humains et la nature. Les Dossiers d'Agropolis International, $\mathrm{N}^{\circ} 13$. Montpellier, Agropolis International, $83 \mathrm{p}$.

Cordier J. B. \& Genin D. 2008 - Pratiques paysannes d'exploitation des arbres et paysages forestiers dans le haut Atlas marocain. Revue forestière française 60(5) : 571-588.

Cornier-Salem M.C, Juhé-Beaulaton D., Boutrais J. \& Roussel B. 2002 - Patrimonialiser la nature tropicale. Dynamiques locales, enjeux internationaux. Paris, IRD (Colloques et séminaires), $467 \mathrm{p}$. 
Davis D.K. 2005 - Potential forests: degradation narratives, science, and environmental policy in protectorate Morocco, 1912-1956. Environmental History 10(2) : 211-238.

Fabiani J.L. 2000 - Éthique et politiques de la techno-nature. À propos de la biologie de la conservation. Revue européenne des sciences sociales, $\mathrm{N}^{\circ}$ XXXVIII-118 : 15-28.

Gadgil M. 2005 - Connaissances écologiques et pratiques de conservation. In Barbault R. (Ed.) Biodiversité, science et gouvernance, Actes de la Conférence Internationale biodiversité, science et gouvernance, Paris, 24-28 janvier 2005 : 163-169.

Gadgil M., Berkes F. \& Folke C. 1993 - Indigenous knowledge for biodiversity conservation. Ambio $22: 151-156$.

Genin D. \& Benchekroun F. (Ed.) 2007 - De la parole aux gestes. Éléments de réflexion sur les dispositifs de gestion concertée des ressources forestières et pastorales au Maroc. Rabat, IRD-France CoopérationENFI, $61 \mathrm{p}$.

Genin D., Fouilleron B. \& Kerautret L. 2012a - Un tempo bien tempéré. Place et rôles des Agdals dans les systèmes d'élevage des Ayt Bouguemmez (Haut Atlas central). In Auclair L. \& Alifriqui M., (Ed.). Agdal : Patrimoine socio-écologique de l'Atlas marocain. Rabat, IRCAM-IRD : 411-434.

Genin D., Kerautret L., Hammi S., Cordier J.B. \& Alifriqui M. 2012b - Biodiversité et pratiques d' Agdal : un élément de l'environnement à l'épreuve de ses fonctions d'utilité pour les sociétés rurales du haut Atlas central. In Auclair L. \& Alifriqui M. (Ed.). Agdal. Patrimoine socio-écologique de l'Atlas marocain. Rabat, Ed IRCAM-IRD : 93-126.

Genin D. \& Simenel R. 2011 - Endogenous management and the shaping of rural forests in southern Morocco: implications for shared forest management options. Human Ecology 39 : 257-269.

Louafi S. \& Tubiana L. 2005 - Conservation et développement. Courrier de la Planète 75 : 4-7.

Migot P. \& Roué M. (Propos recueillis par) 2006 - Robert Barbault ou de l'écologie à la gestion de la biodiversité. Natures Sciences Sociétés 14, Sup. $1: 36-42$.

Ollagnon H. 2000 - La gestion en patrimoine commun de la qualité de l'eau dans un bassin. In Falque M. \& Massenet M. (Ed.), Les ressources en eau. Droits de propriété, économie et environnement. Paris, Dalloz : 325-345.

Parc Naturel Régional du Luberon 2010 - Charte. Objectif 2021. Rapport, Apt, 164 p.

Parc Naturel Régional du Queyras 2010 - Charte 2010-2022, Rapport, Arvieux, 144 p.

Persha L., Agrawal A. \& Chhatre A. 2011 - Social and ecological synergy: local rulemaking, forest livelihood and biodiversity conservation. Science $331: 1606-1608$.

Petit S. \& Fleury Ph. 2010 - Soigner les prairies : vers une réhabilitation des savoirs de la pratique. Terrains \& travaux $17: 41-56$.

Pinton F. 2002 - Manioc et biodiversité : exploration des voies d'un nouveau partenariat. Natures Sciences Sociétés 10(2) : 18-30.

Roué M. 2012 - Histoire et épistémologie des savoirs locaux et autochtones. Revue d'ethnoécologie 1 : 2-14. URL : http://ethnoecologie.revues.org/813 ; DOI : 10.4000/ethnoecologie.813

Roué M. \& Nakashima D. 2005 - Diversité biologique, diversité culturelle : enjeux autour des savoirs locaux. In Barbault R. (Ed.) Biodiversité, science et gouvernance, Actes de la Conférence Internationale, Paris, 24-28 janvier 2005 : 220-222. 
Vincent M. 2011 - Les alpages à l'épreuve du loup. Paris, MSH, Versailles, Quae (Natures sociales), $350 \mathrm{p}$.

\section{NOTES}

1. Dynamiques de la biodiversité en Luberon, formes de connaissance et impacts des pratiques sociales. Programme IFB, 2003 ; Les Agdals du Haut Atlas (Maroc): Biodiversité et gestion communautaire de l'accès aux ressources forestières et pastorales. Programme IFB, 2003-2007; Enjeux des pratiques d'exploitations forestières et pastorales sur les dynamiques forêts-alpages dans deux contextes contrastés de gestion environnementale de part et d'autre du Mont Viso. Programme ECCOREV/CNRS, 2008 ; Politiques publiques et gestion paysannes de l'arbre et de la forêt: dialogue de dupes ou alliance durable. Programme ANR 2008-2010.

2. Mentionnons toutefois que la Charte précise (p. 8) que les actions de conservation de la biodiversité sont envisagées et intégrées dans tous les autres axes de la stratégie de développement du territoire du Parc et que la préoccupation de la biodiversité y est considérée comme transversale et omniprésente.

3. « en bas » est utilisé pour désigner la maison du Parc qui se trouve dans une commune en contrebas de celle où habite la personne interrogée

\section{RÉSUMÉS}

La gestion des ressources naturelles et des territoires peut prendre des formes diverses qui ont largement à voir avec les modes de pensée des acteurs qui les mettent en place. En prenant l'exemple de trois territoires contrastés (Parcs Naturels Régionaux du Luberon et du Queyras dans le sud de la France et Haut Atlas au Maroc), nous nous attacherons ici à montrer comment les approches savantes et populaires diffèrent dans la perception, la définition de normes et le façonnage des territoires, ainsi que dans l'appréhension de la biodiversité. Les zonages proposés des territoires révèlent des différences notables entre gestionnaires institutionnels et populations locales: divisions d'espaces répondant à des problématiques de protection sectorielles d'espèces ou de milieux, ou mises en conformité avec des directives nationales ou internationales de protection répondant à des critères scientifiques d'évaluation des milieux et des espèces d'un côté, critères géographiques, historiques, culturels et économiques, et de complémentarité d'usages d'espaces différenciés de l'autre. De même en ce qui concerne la biodiversité, l'approche savante vise la recherche de lois générales par sectorisation des recherches et du savoir spécialisés sur des éléments ciblés de la biodiversité (une espèce, un milieu), alors que les populations locales insistent sur un «particularisme » local non généralisant, mais dont l'approche est à la fois systémique, mettant en avant des interrelations multiples, et fonctionnaliste par rapport aux activités humaines. Ces différentes approches entraînent souvent des dialogues difficiles, exacerbés par une certaine asymétrie des pouvoirs et une méconnaissance mutuelle de la teneur et des modes de construction des savoirs des groupes d'acteurs en présence.

Natural resources and territories management can take diverse forms, depending on ways of thinking and knowledge of people involved in. Based on examples taken from three contrasted 
areas (Regional Natural Parks of Luberon and Queyras in Southern France, and High Atlas territories in Morocco), we will show to which extent academic and local approaches differ in the ways of perceiving, managing and shaping territories, as well as biodiversity concerns. Territory delimitations and zoning illustrate these discrepancies: divisions of the total area on the basis of specific species or ecosystem protection problematic, or accordance with national or international directives in matter of environmental protection, on the one hand; geographic, historical, cultural and economical criterions, and a generalized concern of seeking functional complementarities of differentiated resources areas uses, on the other hand. Concerning biodiversity, the academic approach aims at defining general rules by optimizing researches and specialized knowledge on specific elements of biodiversity such as a species or an ecosystem, while locals argue forms of local particularisms, away from any generalization, but whose vision combines both systemic and functionalist aspects in relation to human activities. This usually leads to a certain foul's dialogue between these actors, which is exacerbated by a strong power asymetry in matter of formal resource management competencies, and by a certain mutual ignorance concerning the content and the construction modes of these different types of knowledge.

\section{INDEX}

Mots-clés : biodiversité, formes et normes de gestion, savoirs savants, savoirs populaires, Luberon, Queyras, Haut atlas marocain

Keywords : biodiversity, natural resource management forms and norms, academic knowledge, local knowledge, Luberon, Queyras, Moroccan High Atlas

\section{AUTEURS}

\section{CHANTAL ASPE}

Université Aix-Marseille

Laboratoire Population Environnement Développement, UMR IRD 151

3 Place Victor Hugo, 13331 Marseille Cedex 03

chantal.aspe@univ-amu.fr

\section{DIDIER GENIN}

Université Aix-Marseille

Laboratoire Population Environnement Développement, UMR IRD 151

3 Place Victor Hugo, 13331 Marseille Cedex 03

didier.genin@ird.fr 